\title{
Mixed systems with minimal and maximal lifetime variances
}

\author{
Marek Beśka • Krzysztof Jasiński • \\ Tomasz Rychlik • Marcin Spryszyński
}

Received: 11 October 2010 / Published online: 23 April 2011

(C) The Author(s) 2011. This article is published with open access at Springerlink.com

\begin{abstract}
We consider the mixed systems composed of a fixed number of components whose lifetimes are i.i.d. with a known distribution which has a positive and finite variance. We show that a certain of the $k$-out-of- $n$ systems has the minimal lifetime variance, and the maximal one is attained by a mixture of series and parallel systems. The number of the $k$-out-of- $n$ system, and the probability weights of the mixture depend on the first two moments of order statistics of the parent distribution of the component lifetimes. We also show methods of calculating extreme system lifetime variances under various restrictions on the system lifetime expectations, and vice versa.
\end{abstract}

Keywords Mixed system $\cdot k$-out-of- $n$ system $\cdot$ Samaniego signature $\cdot$ Order statistic $\cdot$ i.i.d. random variables $\cdot$ Variance

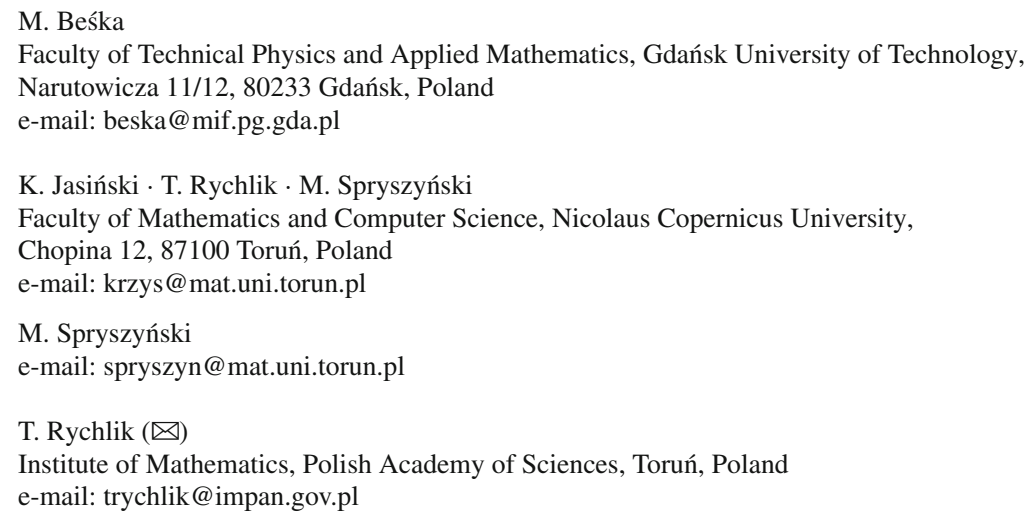




\section{Introduction and auxiliary result}

We consider a coherent system composed of $n$ identical elements. We assume that the non-negative component lifetimes are independent and have identical known distribution function $F$ with a positive and finite variance. Then the distribution function of the system lifetime has the well-known Samaniego representation

$$
\mathbb{P}(T \leq t)=\sum_{i=1}^{n} s_{i} \mathbb{P}\left(X_{i: n} \leq t\right)
$$

where $X_{1: n} \leq \cdots \leq X_{n: n}$ denote the order statistics of component lifetimes. Under the assumptions,

$$
\mathbb{P}\left(X_{i: n} \leq t\right)=\sum_{k=i}^{n} B_{k, n}(F(t)), \quad i=1, \ldots, n
$$

where

$$
B_{k, n}(x)=\left(\begin{array}{l}
n \\
k
\end{array}\right) x^{k}(1-x)^{n-k}, \quad 0 \leq x \leq 1, \quad k=0, \ldots, n,
$$

are the Bernstein polynomials of degree $n$. Vector $\mathbf{s}=\left(s_{1}, \ldots, s_{n}\right)$, called the Samaniego signature, depends on the system structure function $\varphi$ as follows

$$
s_{i}=\frac{1}{n !} \sum_{\pi \in \Pi^{n}} \mathbf{1}_{\{i\}}\left(\max _{1 \leq j \leq n}\left\{j \varphi\left(\sum_{k=j}^{n} \mathbf{e}_{\pi(k)}\right)\right\}\right), \quad i=1, \ldots, n,
$$

where $\Pi^{n}$ stand for the family of all permutations of the set $\{1, \ldots, n\}, \mathbf{1}_{A}$ is the indicator function of set $A$, and $\mathbf{e}_{k}$ denotes the $k$ th standard basis vector in $\mathbb{R}^{n}$ for $k=1, \ldots, n$. Obviously, distribution function (1.2) does not depend on the system structure, and the signature is independent of the component lifetime distribution.

Samaniego (1985) proved representation (1.1) under the condition that $F$ is continuous. Then all the order statistics are different almost surely, and the signature has a natural probabilistic interpretation

$$
s_{i}=\mathbb{P}\left(T=X_{i: n}\right), \quad i=1, \ldots, n .
$$

Navarro et al. (2008) extended (1.1) to the case of arbitrary (possibly discontinuous) exchangeable joint distribution of $X_{1}, \ldots, X_{n}$, and so we drop the assumption of continuity of $F$ here. By (1.3) (cf. also (1.4)), we can check that the Samaniego signature of every system of size $n$ belongs to the simplex

$$
\mathbb{S}^{n}=\left\{\mathbf{s}=\left(s_{1}, \ldots, s_{n}\right) \in \mathbb{R}^{n}: s_{i} \geq 0, \quad i=1, \ldots, n, \quad \sum_{i=1}^{n} s_{i}=1\right\} .
$$


It is difficult to establish all coherent systems with $n$ components, especially when $n$ is large. There are one trivial system with one element, and two systems, series and parallel ones, with two elements. All the systems of sizes 3 and 4 were described in Kochar et al. (1999) and Shaked and Suarez-Llorens (2003), respectively. Navarro and Rubio (2010) presented an algorithm of determining all the systems with arbitrarily fixed number of components. They verified that there are 180 and 16,145 essentially different (up to renumbering of components) systems composed of 5 and 6 items, respectively. The corresponding numbers of signatures are less, because some essentially different systems have identical signatures. There are not known rules describing the distribution of system signatures over the set (1.5). Evidently, the signatures of $k$-out-of- $n$ systems, $k=1, \ldots, n$, correspond to the simplex vertices $\mathbf{e}_{n+1-k}, k=1, \ldots, n$.

Formula (1.1) implies that the lifetime distribution of the coherent system with signature $\mathbf{s}=\left(s_{1}, \ldots, s_{n}\right)$ is identical with the lifetime distribution of randomly chosen among the $(n+1-i)$-out-of- $n$ systems, when the respective choice probabilities are $s_{i}, i=1, \ldots, n$. For mathematical convenience, Boland and Samaniego (2004) introduced the notion of mixed systems. The mixed system of size $n$ with signature $\mathbf{s}=\left(s_{1}, \ldots, s_{n}\right)$ being an arbitrary point of (1.5), is the randomly chosen $(n+1-i)$ out-of- $n$ system, when the choice distribution is represented by the signature $\mathbf{s}$. Navarro et al. (2008) noticed that for every coherent and mixed systems of sizes smaller than $n$ there exists a mixed system with $n$ components and the same lifetime distribution. By (1.1), it is clear that whatever is the exchangeable distribution of the component lifetimes, the shortest expected lifetime among all the mixed systems has the series system, and the parallel one has the longest mean lifetime.

The purpose of this paper is to determine the mixed systems with minimal and maximal lifetime variances under the additional assumptions that the component lifetimes are independent with a known common distribution function $F$. When $F$ has a positive finite variance, we can determine the following positive, finite and strictly increasing sequences

$$
\begin{aligned}
\mu_{i} & =\mathbb{E} X_{i: n}=\int_{0}^{1} F^{-1}(x) n B_{i-1, n-1}(x) d x, \\
\tau_{i} & =\mathbb{E} X_{i: n}^{2}=\int_{0}^{1}\left[F^{-1}(x)\right]^{2} n B_{i-1, n-1}(x) d x, \quad i=1, \ldots, n,
\end{aligned}
$$

where $F^{-1}(x)=\sup \{y: F(y) \leq x\}, 0 \leq x \leq 1$, denotes the the right-continuous upper quantile function of $F$, and $n B_{i-1, n-1}(x), 0 \leq x \leq 1, i=1, \ldots, n$, are the density functions of the consecutive order statistics from the standard uniform i.i.d. sample of size $n$ (see, e.g., Arnold et al. 1992, p. 109). The respective variances are denoted as

$$
\sigma_{i}=\operatorname{Var} X_{i: n}=\tau_{i}-\mu_{i}^{2}, \quad i=1, \ldots, n
$$


Accordingly, for arbitrary $\mathbf{s} \in \mathbb{S}^{n}$ the lifetime $T=T(\mathbf{s})$ of the respective mixed system has the moments

$$
\begin{aligned}
& \mu(\mathbf{s})=\mathbb{E} T(\mathbf{s})=\sum_{i=1}^{n} s_{i} \mu_{i}, \\
& \tau(\mathbf{s})=\mathbb{E} T^{2}(\mathbf{s})=\sum_{i=1}^{n} s_{i} \tau_{i}, \\
& \sigma(\mathbf{s})=\operatorname{Var} T(\mathbf{s})=\sum_{i=1}^{n} s_{i} \tau_{i}-\left(\sum_{i=1}^{n} s_{i} \mu_{i}\right)^{2} .
\end{aligned}
$$

We aim at determining the extreme values of (1.9) as the signatures range over (1.5). When the parent distribution function $F$ is fixed, and so (1.6) and (1.7) are known numbers, this is the classic quadratic programming problem which can be numerically solved by means of standard procedures. Our purpose is to provide more general conclusions valid for various $F$. The crucial tool for our study is the following theorem.

Theorem 1 Let $X_{1}, \ldots, X_{n}, n \geq 3$, be i.i.d. random variables with a common distribution function such that $0<\operatorname{Var} X_{1}<\infty$. Then, under notation (1.6) and (1.7), the sequence

$$
d(F, n ; k)=\frac{\tau_{k+1}-\tau_{k}}{\mu_{k+1}-\mu_{k}}, \quad k=1, \ldots, n-1,
$$

is increasing.

In Sect. 2, we show that the minimal lifetime variance has one of $k$-out-of- $n$ systems, and apply Theorem 1 for proving that the lifetime variance is maximized for a random mixture of the series ans parallel systems. The number of the $k$-out-of- $n$ system and the mixture coefficients depend on the parent distribution function $F$. Final results are specified for the exemplary cases of uniformly and exponentially distributed component lifetimes. In Sect. 3, we analyze bounds for the system lifetime variances under restrictions on the first lifetime moment and vice versa. For instance, we find the mixed systems with the minimal lifetime variance among the ones whose lifetime expectations are not less than a prescribed minimal level. We also determine the maximal lifetime means under an upper constraint on the variance. Such problems are relevant in practice, and can be solved once we specify the component lifetime distribution. Section 4 contains the proof of Theorem 1.

It is worth noting that Theorem 1 holds true for arbitrary non-degenerate distribution functions $F$ with finite second moments. It follows that all the results of Sects. 2 and 3 could be stated in a more general form. Namely, instead of the mixed system lifetime $T(\mathbf{s})$, we could write the order statistic $X_{I: n}$ with random index $I$ that is independent of original variables $X_{1}, \ldots, X_{n}$, and has the distribution $\mathbb{P}(I=i)=s_{i}, i=1, \ldots, n$, determined by the signature. Then the assumption that the support of $F$ is contained in the non-negative half-axis, natural in the reliability problems, may be dropped. 
As far as we know, the problems of evaluating lifetime variances for arbitrary mixed systems composed of items with a fixed lifetime distribution have not been treated in the literature by now. There are known some lifetime variance estimates for systems with fixed structure and varying distributions of component lifetimes. Papadatos (1995) provided sharp upper bounds for the variances of order statistics (and so $k$-out-of- $n$ system lifetimes) coming from i.i.d. populations with an arbitrary parent distribution, expressed in the distribution variance units. The bounds were refined by Papadatos (1997) and Jasiński and Rychlik (2011) in the case of symmetrically distributed random variables. Jasiński et al. (2009) extended the results of Papadatos (1995) to the case of arbitrarily fixed mixed systems. Rychlik (1994) described methods of calculating sharp lifetime variance bounds for $k$-out-of- $n$ systems built of exchangeable components with a known marginal lifetime distribution. The results were extended in Rychlik (2011) to a slightly wider family of mixed systems with all the signature coordinates identical except for one. Also Rychlik (2008), provided sharp non-parametric lower and upper bounds for the lifetime variances of $k$-out-of- $n$ systems composed of exchangeable elements, gauged in the single component lifetime variance units.

There are also known few bounds for the mixed central moments of order two for the order statistics. An optimal inequality for the covariance of the smaller and greater of two i.i.d. random variables expressed in the population variance units was determined by Papathanasiou (1990) (see also López-Blázquez and Salamanca-Miño 1999). This was sharpened for the finite support populations by López-Blázquez and Salamanca-Miño (1999), and generalized to dependent samples by Balakrishnan and Balasubramanian (1993). A similar inequality for the covariance of the minimum and maximum of three i.i.d. random variables was provided by Papadatos (1999). Sharp bounds for the correlation of the minimum and maximum of two i.i.d. variables from general and finite support populations were established by Terrel (1983) and López-Blázquez and Salamanca-Miño (1999), respectively. The former evaluation was generalized by Székely and Móri (1985) to the case of general functions of arbitrary two order statistics from the i.i.d. sample of any size. Castaño-Martinez et al. (2007) proved that the Székely-Móri bounds hold for the sampling without replacement models as well. Various estimates of the correlation of the smaller and greater of two random variables with arbitrary joint distributions ca be found in Navarro and Balakrishnan (2010). On the other hand, there were established numerous sharp evaluations of lifetime expectations of various mixed systems (and especially $k$-out-of- $n$ ones) under diverse assumptions on the marginal and joint distributions of the component lifetimes, but we do not cite them here.

\section{Systems with extreme lifetime variances}

Theorem 2 Let $T(\mathbf{s})$ be the lifetime of a mixed system with signature $\mathbf{s}=$ $\left(s_{1}, \ldots, s_{n}\right) \in \mathbb{S}^{n}$, composed of $n$ elements with i.i.d. lifetimes whose common distribution has a positive and finite variance. Then, under notation (1.6) and (1.8), the following inequalities are sharp 


$$
\begin{aligned}
& \min _{1 \leq i \leq n} \sigma_{i} \leq \operatorname{Var} T(\mathbf{s}) \\
\leq & \begin{cases}\sigma_{1}, & \text { if } \sigma_{1}-\sigma_{n} \geq\left(\mu_{n}-\mu_{1}\right)^{2}, \\
\sigma_{n}, & \text { if } \sigma_{n}-\sigma_{1} \geq\left(\mu_{n}-\mu_{1}\right)^{2}, \\
\frac{\left(\mu_{n}-\mu_{1}\right)^{2}}{4}+\frac{1}{4}\left(\frac{\sigma_{n}-\sigma_{1}}{\mu_{n}-\mu_{1}}\right)^{2}+\frac{\sigma_{1}+\sigma_{n}}{2}, & \text { if }\left|\sigma_{n}-\sigma_{1}\right|<\left(\mu_{n}-\mu_{1}\right)^{2} .\end{cases}
\end{aligned}
$$

If the minimum is equal to $\sigma_{k}$ for some $k=1, \ldots, n$, then the lower bound is attained by the $(n+1-k)$-out-of-n system. The upper bounds are attained in the consecutive cases by the series and parallel systems, and the mixture of the series and parallel systems with probabilities $s_{1}=\frac{1}{2}\left[1-\frac{\sigma_{n}-\sigma_{1}}{\left(\mu_{n}-\mu_{1}\right)^{2}}\right]$ and $s_{n}=1-s_{1}$, respectively.

Obviously the above evaluations are invariant under translations, and equivariant under scale transformations. We point out here that the lower bounds of Theorem 2 are attained by proper coherent systems, i.e. the $k$-out-of- $n$ ones. The upper ones are attained by mixtures of the series and parallel systems, and except for the trivial mixtures with either $s_{1}=1$ or $s_{n}=1$, there do not exist coherent systems with signatures $\left(s_{1}, 0, \ldots, 0,1-s_{1}\right)$ for $0<s_{1}<1$. So it is still an open and challenging problem to establish upper, possibly sharp bounds for the lifetime variances of coherent systems.

Proof of Theorem 2 The proof of the lower bound consists in minimizing the concave function (1.9) over the convex, closed and bounded set (1.5). We have

$$
\sigma\left(\alpha \mathbf{s}_{1}+(1-\alpha) \mathbf{s}_{2}\right) \geq \alpha \sigma\left(\mathbf{s}_{1}\right)+(1-\alpha) \sigma\left(\mathbf{s}_{2}\right) \geq \min \left\{\sigma\left(\mathbf{s}_{1}\right), \sigma\left(\mathbf{s}_{2}\right)\right\}
$$

for arbitrary $\mathbf{s}_{1}, \mathbf{s}_{2} \in \mathbb{S}^{n}$ and $0 \leq \alpha \leq 1$. Therefore

$$
\sigma(\mathbf{s})=\sigma\left(\sum_{i=1}^{n} s_{i} \mathbf{e}_{i}\right) \geq \min _{1 \leq i \leq n} \sigma\left(\mathbf{e}_{i}\right)=\min _{1 \leq i \leq n} \sigma_{i}
$$

for all $\mathbf{s} \in \mathbb{S}^{n}$.

For proving the latter inequality, we consider the planar representations of the first two raw moments of the system lifetimes

$$
M(\mathbf{s})=\left(\mathbb{E} T(\mathbf{s}), \mathbb{E} T^{2}(\mathbf{s})\right)=\left(\sum_{i=1}^{n} s_{i} \mu_{i}, \sum_{i=1}^{n} s_{i} \tau_{i}\right), \quad \mathbf{s} \in \mathbb{S}^{n}
$$

In particular $M\left(\mathbf{e}_{i}\right)=\left(\mu_{i}, \tau_{i}\right), i=1, \ldots, n$. The image of the whole simplex (1.5) in the moment transformation $M\left(\mathbb{S}^{n}\right)=\left\{M(\mathbf{s}): \mathbf{s} \in \mathbb{S}^{n}\right\}$ is the convex hull spread over the extreme points $M\left(\mathbf{e}_{i}\right), i=1, \ldots, n$. It follows from Theorem 1 that this is a polygon whose bottom is composed of the line segments joining consecutive points $M\left(\mathbf{e}_{k}\right)$ and $M\left(\mathbf{e}_{k+1}\right), k=1, \ldots, n-1$. The polygon is bounded above by the edge 
joining $M\left(\mathbf{e}_{1}\right)$ and $M\left(\mathbf{e}_{n}\right)$. Accordingly,

$$
\begin{gathered}
M\left(\mathbb{S}^{n}\right)=\left\{(\mu, \tau): \quad \tau_{k}+\frac{\tau_{k+1}-\tau_{k}}{\mu_{k+1}-\mu_{k}}\left(\mu-\mu_{k}\right) \leq \tau \leq \tau_{1}+\frac{\tau_{n}-\tau_{1}}{\mu_{n}-\mu_{1}}\left(\mu-\mu_{1}\right),\right. \\
\left.\mu_{k} \leq \mu \leq \mu_{k+1}, \quad k=1, \ldots, n-1\right\} .
\end{gathered}
$$

In the case $n=2$, the set reduces to a line segment. We can further define

$$
\begin{gathered}
\Sigma\left(\mathbb{S}^{n}\right)=\left\{(\mathbb{E} T(\mathbf{s}), \operatorname{Var} T(\mathbf{s})): \mathbf{s} \in \mathbb{S}^{n}\right\}=\left\{\left(\mu, \tau-\mu^{2}\right):(\mu, \tau) \in M\left(\mathbb{S}^{n}\right)\right\} \\
=\left\{(\mu, \sigma): \tau_{k}+\frac{\tau_{k+1}-\tau_{k}}{\mu_{k+1}-\mu_{k}}\left(\mu-\mu_{k}\right)-\mu^{2} \leq \sigma \leq \tau_{1}+\frac{\tau_{n}-\tau_{1}}{\mu_{n}-\mu_{1}}\left(\mu-\mu_{1}\right)\right. \\
\left.-\mu^{2}, \quad \mu_{k} \leq \mu \leq \mu_{k+1}, k=1, \ldots, n-1\right\} .
\end{gathered}
$$

It is easily seen that among all the systems with an arbitrarily fixed lifetime expectation $\mu_{1} \leq \mu \leq \mu_{n}$, the maximal variance $\tau_{1}+\frac{\tau_{n}-\tau_{1}}{\mu_{n}-\mu_{1}}\left(\mu-\mu_{1}\right)-\mu^{2}$ is attained by the combination of series and parallel systems with probabilities $\alpha=\frac{\mu_{n}-\mu}{\mu_{n}-\mu_{1}}$ and $1-\alpha=\frac{\mu-\mu_{1}}{\mu_{n}-\mu_{1}}$, respectively. The global variance maximum is derived by maximizing

$$
\varsigma(\alpha)=\sigma\left(\alpha \mathbf{e}_{1}+(1-\alpha) \mathbf{e}_{n}\right)=\alpha \tau_{1}+(1-\alpha) \tau_{n}-\left[\alpha \mu_{1}+(1-\alpha) \mu_{n}\right]^{2}, \quad 0 \leq \alpha \leq 1
$$

This is a quadratic concave function that has the global maximum at

$$
\alpha_{*}=\frac{1}{2}\left[1-\frac{\sigma_{n}-\sigma_{1}}{\left(\mu_{n}-\mu_{1}\right)^{2}}\right]
$$

Under restriction to the unit interval, there are possible three cases. In the first case $\alpha_{*} \geq 1$ (i.e., when $\left.\sigma_{1}-\sigma_{n} \geq\left(\mu_{n}-\mu_{1}\right)^{2}\right)$, the restricted maximum equals to $\varsigma(1)=$ $\sigma\left(\mathbf{e}_{1}\right)=\sigma_{1}$. In the second one for $\alpha_{*} \leq 0$ (i.e., for $\left.\sigma_{n}-\sigma_{1} \geq\left(\mu_{n}-\mu_{1}\right)^{2}\right)$, the maximum is $\varsigma(0)=\sigma\left(\mathbf{e}_{n}\right)=\sigma_{n}$. Otherwise the local maximum coincides with the global one and amounts to

$$
\varsigma\left(\alpha_{*}\right)=\sigma\left(\alpha_{*} \mathbf{e}_{1}+\left(1-\alpha_{*}\right) \mathbf{e}_{n}\right)=\frac{\left(\mu_{n}-\mu_{1}\right)^{2}}{4}+\frac{1}{4}\left(\frac{\sigma_{n}-\sigma_{1}}{\mu_{n}-\mu_{1}}\right)^{2}+\frac{\sigma_{1}+\sigma_{n}}{2} .
$$

This completes the proof.

From the above proof we can immediately deduce the following constrained extremes. 
Corollary 1 Under the assumptions of Theorem 2,

$$
\begin{gathered}
\min _{\mathbb{E} T(\mathbf{s})=\mu} \operatorname{Var} T(\mathbf{s})=\tau_{k}+\frac{\tau_{k+1}-\tau_{k}}{\mu_{k+1}-\mu_{k}}\left(\mu-\mu_{k}\right)-\mu^{2}, \\
\mu_{k} \leq \mu \leq \mu_{k+1}, k=1, \ldots, n-1, \\
\max _{\mathbb{E} T(\mathbf{s})=\mu} \operatorname{Var} T(\mathbf{s})=\tau_{1}+\frac{\tau_{n}-\tau_{1}}{\mu_{n}-\mu_{1}}\left(\mu-\mu_{1}\right)-\mu^{2}, \quad \mu_{1} \leq \mu \leq \mu_{n} .
\end{gathered}
$$

The above extreme variances are attained by the mixture systems with signatures $\frac{\mu_{k+1}-\mu}{\mu_{k+1}-\mu_{k}} \mathbf{e}_{k}+\frac{\mu-\mu_{k}}{\mu_{k+1}-\mu_{k}} \mathbf{e}_{k+1}$ and $\frac{\mu_{n}-\mu}{\mu_{n}-\mu_{1}} \mathbf{e}_{1}+\frac{\mu-\mu_{1}}{\mu_{n}-\mu_{1}} \mathbf{e}_{n}$, respectively.

Formula (2.3) provides another proof of the first inequality in (2.1). Indeed, referring to the concavity arguments, we can write

$$
\min _{\mu_{1} \leq \mathbb{E} T(\mathbf{s}) \leq \mu_{n}} \operatorname{Var} T(\mathbf{s})=\min _{1 \leq k \leq n-1} \min _{\mu_{k} \leq \mathbb{E} T(\mathbf{s}) \leq \mu_{k+1}} \operatorname{Var} T(\mathbf{s})=\min _{1 \leq k \leq n-1} \min \left\{\sigma_{k}, \sigma_{k+1}\right\} .
$$

Corollary 2 If $\sigma_{1}=\sigma_{n}$ (in particular, if the distribution function $F$ is symmetric), then we have

$$
\operatorname{Var} T(\mathbf{s}) \leq \frac{\left(\mu_{n}-\mu_{1}\right)^{2}}{4}+\sigma_{1}
$$

with the equality attained for the system with signature $\frac{1}{2}\left(\mathbf{e}_{1}+\mathbf{e}_{n}\right)$.

Example 1 Consider the systems whose component lifetimes have the standard uniform distribution. Then

$$
\begin{aligned}
\mu_{i} & =\frac{i}{n+1}, \\
\sigma_{i} & =\frac{i(n+1-i)}{(n+1)^{2}(n+2)}, \quad i=1, \ldots, n,
\end{aligned}
$$

(cf. Johnson et al. 1995, p. 280). By Theorem 2,

$$
\min _{\mathbf{s} \in \mathbb{S}^{n}} \operatorname{Var} T(\mathbf{s})=\sigma_{1}=\sigma_{n}=\frac{n}{(n+1)^{2}(n+2)},
$$

which tends to 0 at the rate $\mathcal{O}\left(n^{-2}\right)$ as $n$ increases to infinity. Due to Corollary 2, we have

$$
\max _{\mathbf{s} \in \mathbb{S}^{n}} \operatorname{Var} T(\mathbf{s})=\sigma\left(\frac{\mathbf{e}_{1}+\mathbf{e}_{n}}{2}\right)=\frac{1}{4}-\frac{n}{(n+1)(n+2)} .
$$

As the system size increases to infinity, the variances tend to $\frac{1}{4}$ which is the variance of the symmetric two-point distribution on 0 and 1 . This is the maximal variance of distributions with the support contained in any interval of length 1 . We checked that 
among all the coherent systems of sizes $n=3,4,5$, the maxima are attained for the systems with lifetimes $T_{c}=\min \left\{X_{1}, \max \left\{X_{2}, \ldots, X_{n}\right\}\right\}$ and for their duals with lifetimes $T_{d}=\max \left\{X_{1}, \min \left\{X_{2}, \ldots, X_{n}\right\}\right\}$. The respective Samaniego signatures are $\mathbf{s}_{c}=\left(\frac{1}{n}, \ldots, \frac{1}{n}, \frac{2}{n}, 0\right)$ and $\mathbf{s}_{d}=\left(0, \frac{2}{n}, \frac{1}{n}, \ldots, \frac{1}{n}\right)$. They have lifetime variances

$$
\operatorname{Var} T\left(\mathbf{s}_{c}\right)=\operatorname{Var} T\left(\mathbf{s}_{d}\right)=\frac{1}{12}-\frac{2}{(n+1)(n+2)}+\frac{1}{n(n+1)}-\frac{1}{n^{2}(n+1)^{2}}
$$

which tend to the lifetime variance of a single component, equal to $\frac{1}{12}$, as $n$ increases. We can not guarantee though, that the systems with such structures have maximal lifetime variances for all $n \geq 6$.

Example 2 Take the systems whose components have i.i.d. lifetimes with the standard exponential distribution. Hence

$$
\begin{aligned}
\mu_{k} & =\sum_{i=n+1-k}^{n} \frac{1}{i}, \\
\sigma_{k} & =\sum_{i=n+1-k}^{n} \frac{1}{i^{2}}, \quad i=1, \ldots, n,
\end{aligned}
$$

(cf. Johnson et al. 1994, p. 500). It follows that

$$
\min _{\mathbf{s} \in \mathbb{S}^{n}} \operatorname{Var} T(\mathbf{s})=\sigma_{1}=\frac{1}{n^{2}} .
$$

Since

$$
\left|\sigma_{n}-\sigma_{1}\right|=\sum_{i=1}^{n-1} \frac{1}{i^{2}}<\sum_{i=1}^{n-1} \frac{1}{i^{2}}+\sum_{1 \leq i<j \leq n-1} \frac{2}{i j}=\left(\sum_{i=1}^{n-1} \frac{1}{i}\right)^{2}=\left(\mu_{n}-\mu_{1}\right)^{2},
$$

by Theorem 2

$$
\max _{\mathbf{s} \in \mathbb{S}^{n}} \operatorname{Var} T(\mathbf{s})=\frac{1}{4}\left(\sum_{i=1}^{n-1} \frac{1}{i}\right)^{2}+\frac{1}{2} \sum_{i=1}^{n-1} \frac{1}{i^{2}}+\frac{1}{4}\left(\frac{\sum_{i=1}^{n-1} \frac{1}{i^{2}}}{\sum_{i=1}^{n-1} \frac{1}{i}}\right)^{2}+\frac{1}{n^{2}} .
$$

It is attained by the combination of the series and parallel systems with coefficients

$$
s_{1}=\frac{1}{2}\left[1-\frac{\sum_{i=1}^{n-1} \frac{1}{i^{2}}}{\left(\sum_{i=1}^{n-1} \frac{1}{i}\right)^{2}}\right]
$$

and $s_{n}=1-s_{1}$, respectively. If $n$ tends to infinity, then $s_{1} \nearrow \frac{1}{2} \swarrow s_{n}$ whereas (2.5) increase to infinity at the rate $\mathcal{O}\left(\ln ^{2} n\right)$. On the other hand, the maximal lifetime 
variances among all coherent systems of sizes up to $n=5$ are attained by the parallel ones. The lifetime variances of the parallel systems asymptotically approach the finite number $\frac{\pi^{2}}{6}$. We do not claim that the parallel systems have maximal lifetime variances among all the coherent systems of sizes $n \geq 6$.

Example 3 Consider now the systems composed of elements with independent lifetimes that have the Pareto distribution function $F_{\theta}(t)=1-t^{-\theta}, t \geq 1$, for some $\theta>2$. Then

$$
\begin{aligned}
& \mu_{i}(\theta)=\mathbb{E}_{\theta} X_{i: n}=\prod_{k=n+1-i}^{n} \frac{k \theta}{k \theta-1}, \\
& \tau_{i}(\theta)=\mathbb{E}_{\theta} X_{i: n}^{2}=\prod_{k=n+1-i}^{n} \frac{k \theta}{k \theta-2}, \quad i=1, \ldots, n,
\end{aligned}
$$

(cf. Johnson et al. 1994, p. 599). We show that for every integer $n \geq 2$ there exists $\theta(n)$ such that for all $2<\theta<\theta(n)$

$$
\max _{\mathbf{s} \in \mathbb{S}^{n}} \operatorname{Var}_{\theta} T(\mathbf{s})=\sigma_{n}(\theta)=\tau_{n}(\theta)-\mu_{n}^{2}(\theta) .
$$

Observe that

$$
\begin{aligned}
& \lim _{\theta \searrow 2}\left[\tau_{n}(\theta)-\tau_{1}(\theta)-2 \mu_{n}^{2}(\theta)\right] \\
= & \lim _{\theta \searrow 2}\left[\frac{\theta}{\theta-2} \prod_{k=2}^{n} \frac{k \theta}{k \theta-2}-\frac{n \theta}{n \theta-2}-2\left(\prod_{k=1}^{n} \frac{k \theta}{k \theta-1}\right)^{2}\right]=+\infty .
\end{aligned}
$$

In consequence, there is $\theta(n)>2$ such that

$$
\begin{aligned}
\sigma_{n}(\theta)-\sigma_{1}(\theta)-\left[\mu_{n}(\theta)-\mu_{1}(\theta)\right]^{2} & =\tau_{n}(\theta)-\tau_{1}(\theta)-2 \mu_{n}(\theta)\left[\mu_{n}(\theta)-\mu_{1}(\theta)\right] \\
& >\tau_{n}(\theta)-\tau_{1}(\theta)-2 \mu_{n}^{2}(\theta)>0
\end{aligned}
$$

for all $2<\theta<\theta(n)$. Combining (2.7) with (2.1), we obtain (2.6), as claimed. The same conclusion holds for the modified Pareto distributions $G_{\theta}(t)=F_{\theta}(t+1)=$ $1-(t+1)^{-\theta}, t>0, \theta>2$, which is more frequently used in the reliability studies. Indeed, the means and variances of the respective order statistics satisfy $v_{i}(\theta)=$ $\mu_{i}(\theta)-1, \varsigma_{i}(\theta)=\sigma_{i}(\theta), i=1, \ldots, n$. Therefore for every $n$ and $2<\theta<\theta(n)$ we have

$$
\varsigma_{n}(\theta)-\varsigma_{1}(\theta)-\left[v_{n}(\theta)-v_{1}(\theta)\right]^{2}=\sigma_{n}(\theta)-\sigma_{1}(\theta)-\left[\mu_{n}(\theta)-\mu_{1}(\theta)\right]^{2}>0,
$$

which implies that the parallel system has the maximal lifetime variance.

Similarly, one can construct lifetime distributions of components such that the series system has the maximal lifetime variance. For instance, one can take the negative 
Pareto distributions $F_{\theta}(t)=(-t)^{-\theta}, t \leq-1$, with $\theta$ slightly exceeding 2 , truncate them on the left at a large negative level, and shift them to the positive half-axis. This means that there are component lifetime distributions for which maximal system lifetime variances are attained by proper coherent systems, either parallel or series ones, and no formula is redundant in (2.1).

\section{Constrained extremes}

Analyzing inequalities describing the moment set (2.2), it is possible to present further constrained evaluations of the mean and variance of the mixed system lifetimes, different from the ones presented in Corollary 1. However, the shape of (2.2) depends on the moments of order statistics of component lifetime distribution function, and evaluations can be established under specification of the parent distribution.

For instance, for the standard uniform component distribution (2.2) has the form

$$
\begin{aligned}
\Sigma\left(\mathbb{S}^{n}\right)=\{ & (\mu, \sigma): 2 \frac{k+1}{n+2} \mu-\frac{k(k+1)}{(n+1)(n+2)}-\mu^{2} \leq \sigma \leq \mu-\frac{n}{(n+1)(n+2)} \\
& \left.-\mu^{2}, \quad \frac{k}{n+1} \leq \mu \leq \frac{k+1}{n+1}, \quad k=1, \ldots, n-1\right\}
\end{aligned}
$$

where $\mu$ and $\sigma$ denote the lifetime mean and variance of an arbitrarily fixed mixed system composed of $n$ items with independent and standard uniform lifetimes. Each parabola composing the lower envelope is first increasing and then decreasing, and has the maximum at $\frac{k+1}{n+2} \in\left(\mu_{k}, \mu_{k+1}\right)=\left(\frac{k}{n+1}, \frac{k+1}{n+1}\right)$. The upper bound is also increasing-decreasing, and symmetric about $\frac{1}{2}$. Exemplary graphical representation of (3.1) for $n=5$ is given in Fig. 1 .

We can easily check that

$$
\max _{\operatorname{Var} T(\mathbf{s})=\sigma} \mathbb{E} T(\mathbf{s})=\frac{1}{2}+\left(\frac{1}{4}-\frac{n}{(n+1)(n+2)}-\sigma\right)^{1 / 2},
$$

for all $\sigma_{1}=\frac{n}{(n+1)^{2}(n+2)} \leq \sigma \leq \varsigma\left(\alpha_{*}\right)=\frac{1}{4}-\frac{n}{(n+1)(n+2)}$, and the maximum is attained by the mixture of the series and parallel systems with coefficients $s_{1}$ and $s_{n}$ defined as

$$
s_{1}=\frac{n+1}{n-1}\left[\frac{n}{n+1}-\frac{1}{2}-\left(\frac{1}{4}-\frac{n}{(n+1)(n+2)}-\sigma\right)^{1 / 2}\right]<\frac{1}{2}<s_{n}=1-s_{1} \text {. }
$$

The formula for the respective minimum differs from (3.2) only by putting minus instead of plus in front of the square root expression, and it is attained by the dual system with the interchanged weights of the series and parallel systems. The estimates remain valid if we replace the variance condition by a less stringent one $\operatorname{Var} T(\mathbf{s}) \geq \sigma$. 


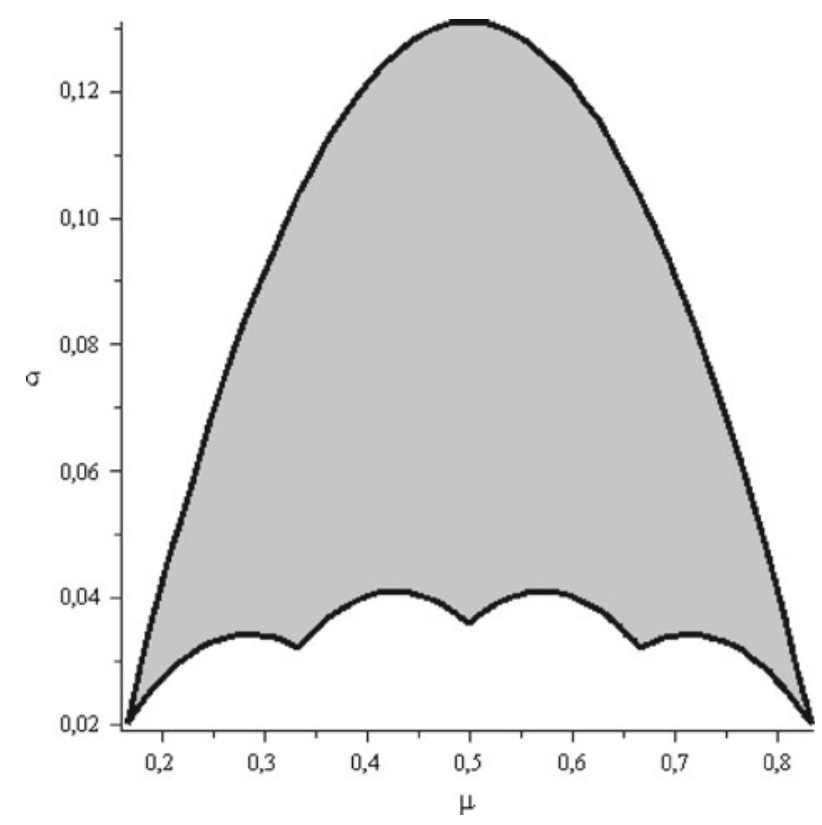

Fig. 1 Set $\Sigma\left(\mathcal{S}^{5}\right)$ for uniform standard distribution

Clearly, for all possible $\sigma$ we have

$$
\begin{aligned}
\min _{\operatorname{Var} T(\mathbf{s}) \leq \sigma} \mathbb{E} T(\mathbf{s})=\mu_{1} & =\frac{1}{n+1}, \\
\max _{\operatorname{Var} T(\mathbf{s}) \leq \sigma} \mathbb{E} T(\mathbf{s})=\mu_{n} & =\frac{n}{n+1} .
\end{aligned}
$$

Similarly, for all $\mu_{1}=\frac{1}{n+1} \leq \mu \leq \mu_{n}=\frac{n}{n+1}$ yields

$$
\min _{\mathbb{E} T(\mathbf{s}) \leq \mu} \operatorname{Var} T(\mathbf{s})=\sigma_{1}=\min _{\mathbb{E} T(\mathbf{s}) \geq \mu} \operatorname{Var} T(\mathbf{s})=\sigma_{n}=\frac{n}{(n+1)^{2}(n+2)} .
$$

We can also easily determine the maximal system lifetime variances under the inequality restrictions on the respective mean. These are either the global maxima $\max _{\mathbf{s} \in \mathbb{S}^{n}} \operatorname{Var} T(\mathbf{s})$ (see (2.4)) or the restricted maximum $\max _{\mathbb{E} T(\mathbf{s})=\mu} \operatorname{Var} T(\mathbf{s})$ (see (3.1)) for the extreme value of admissible mean $\mu$, and the choice depends on the relation between $\mu$ and global maximum point $\frac{1}{2}$. For the practice purposes, the most important are descriptions of systems with maximal lifetime mean under upper restrictions on the respective variance, and ones with minimal variance when a condition on minimal lifetime expectation is imposed. 
In the standard exponential case, the admissible pairs of means and variances satisfy the relations

$$
\begin{aligned}
\sum_{i=n-k}^{n} \frac{1}{i^{2}}-\left(\sum_{i=n-k}^{n} \frac{1}{i}-\mu\right)^{2} \leq \sigma & \leq\left(\sum_{i=1}^{n-1} \frac{1}{i}+\frac{\sum_{i=1}^{n-1} \frac{1}{i^{2}}}{\sum_{i=1}^{n-1} \frac{1}{i}}+\frac{2}{n}\right) \mu \\
& -\mu^{2}-\frac{1}{n}\left(\sum_{i=1}^{n-1} \frac{1}{i}+\frac{\sum_{i=1}^{n-1} \frac{1}{i^{2}}}{\sum_{i=1}^{n-1} \frac{1}{i}}\right), \\
\text { for } \sum_{i=n-k+1}^{n} \frac{1}{i} \leq \mu & \leq \sum_{i=n-k}^{n} \frac{1}{i}, \quad k=1, \ldots, n-1 .
\end{aligned}
$$

The lower bound consists of increasing concave quadratic functions with global maxima attained at the right-hand ends $\mu_{k+1}=\sum_{i=n-k}^{n} \frac{1}{i}, k=1, \ldots, n-1$, of their domains. The upper one is concave quadratic with the maximum presented in (2.5) for

$$
\mu_{*}=\mu_{n}-\alpha_{*}\left(\mu_{n}-\mu_{1}\right)=\frac{1}{2}\left(\sum_{i=1}^{n-1} \frac{1}{i}+\frac{\sum_{i=1}^{n-1} \frac{1}{i^{2}}}{\sum_{i=1}^{n-1} \frac{1}{i}}+\frac{2}{n}\right) .
$$

The exemplary set $\Sigma\left(\mathbb{S}^{5}\right)$ is presented in Fig. 2 .

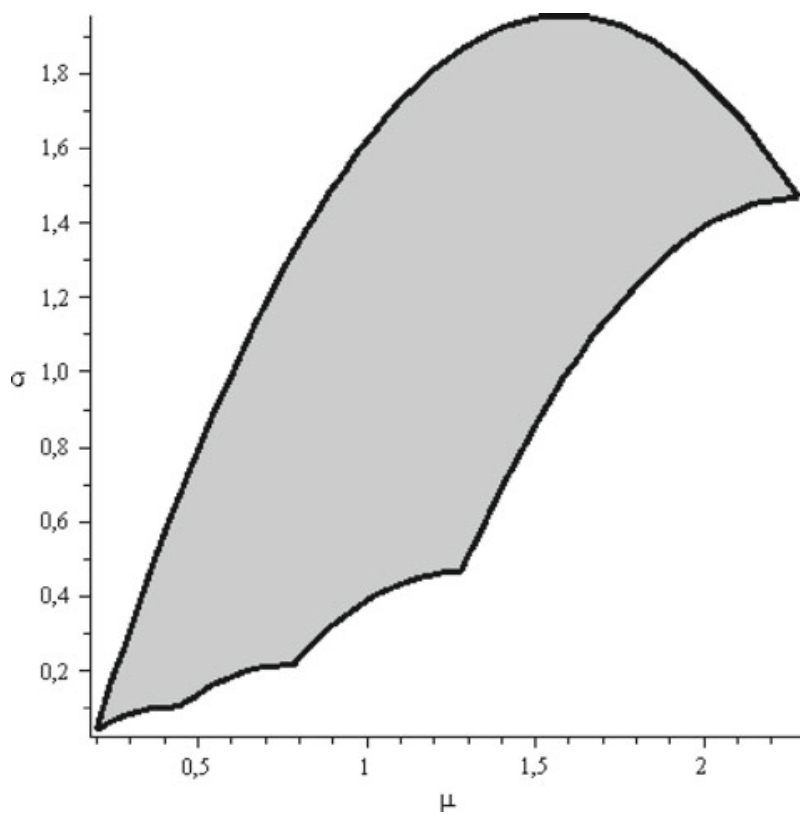

Fig. 2 Set $\Sigma\left(\mathcal{S}^{5}\right)$ for exponential standard distribution 
We immediately see that $\min _{\mathbb{E}} T(\mathbf{s}) \geq \mu \operatorname{Var} T(\mathbf{s})$ amounts to the lower bound in (3.3). Moreover, $\max _{\mathbb{E} T(\mathbf{s}) \geq \mu} \operatorname{Var} T(\mathbf{s})$ coincides with the global maximum described in (2.5) when $\mu \leq \mu_{*}$ and the upper bound in (3.3) otherwise. We also have

$$
\max _{\operatorname{Var} T(\mathbf{s})=\sigma} \mathbb{E} T(\mathbf{s})=\sum_{i=n-k}^{n} \frac{1}{i}-\left(\sum_{i=n-k}^{n} \frac{1}{i^{2}}-\sigma\right)^{\frac{1}{2}},
$$

when $\sigma_{k}=\sum_{i=n-k+1}^{n} \frac{1}{i^{2}} \leq \sigma \leq \sigma_{k+1}=\sum_{i=n-k}^{n} \frac{1}{i^{2}}, k=1, \ldots, n-1$, and

$\max _{\operatorname{Var} T(\mathbf{s})=\sigma} \mathbb{E} T(\mathbf{s})=\frac{1}{2}\left[\sum_{i=1}^{n-1} \frac{1}{i}+\frac{\sum_{i=1}^{n-1} \frac{1}{i^{2}}}{\sum_{i=1}^{n-1} \frac{1}{i}}+\frac{2}{n}+\left(\sum_{i=1}^{n-1} \frac{1}{i}+\frac{\sum_{i=1}^{n-1} \frac{1}{i^{2}}}{\sum_{i=1}^{n-1} \frac{1}{i}}+\frac{4}{n}-4 \sigma\right)^{\frac{1}{2}}\right]$,

when $\sigma_{n}=\sum_{i=1}^{n} \frac{1}{i^{2}} \leq \sigma \leq \varsigma\left(\alpha_{*}\right)=\frac{1}{4}\left(\sum_{i=1}^{n-1} \frac{1}{i}\right)^{2}+\frac{1}{4}\left(\frac{\sum_{i=1}^{n-1} \frac{1}{i^{2}}}{\sum_{i=1}^{n-1} \frac{1}{i}}\right)^{2}+\frac{1}{2} \sum_{i=1}^{n-1} \frac{1}{i^{2}}+\frac{1}{n^{2}}$. If we replace the condition by $\operatorname{Var} T(\mathbf{s}) \leq \sigma$, we get the bound of (3.4) when $\sigma<$ $\sigma_{n}=\sum_{i=1}^{n} \frac{1}{i^{2}}$, and $\mu_{n}=\sum_{i=1}^{n} \frac{1}{i}$ otherwise. It is an elementary task to calculate the signatures of mixed systems attaining the bounds, and we omit presenting the solutions here.

\section{Proof of Theorem 1}

We first prove an auxiliary result.

Lemma 1 Let $f, h:(0,1) \mapsto \mathbb{R}$ be integrable with respect to a non-decreasing non-constant right-continuous function $\mu:(0,1) \mapsto \mathbb{R}$. If they satisfy

$$
\int_{0}^{1} f(x) \mu(d x)=\int_{0}^{1} h(x) \mu(d x)
$$

and

$$
[f(x)-h(x)]\left(x-x_{0}\right)>0 \quad \mu-a . e .
$$

for some $0<x_{0}<1$, then inequality

$$
\int_{0}^{1} l(x) f(x) \mu(d x)>\int_{0}^{1} l(x) h(x) \mu(d x)
$$

holds for arbitrary non-decreasing, non-constant $\mu$-a.e, and right-continuous function $l:(0,1) \mapsto \mathbb{R}$ under the condition that both integrals exist and are finite. 
Proof We can write condition (4.1) as

$$
\int_{x_{0}}^{1}[f(x)-h(x)] \mu(d x)=\int_{0}^{x_{0}}[h(x)-f(x)] \mu(d x) .
$$

By (4.2), both the differences are positive $\mu$-a.e. on the respective intervals. The integrals are positive as well, because so is their sum. If $l$ satisfies the assumptions, we have

$$
\begin{aligned}
\int_{0}^{x_{0}}\left[l(x)-l\left(x_{0}\right)\right][h(x)-f(x)] \mu(d x) & \leq\left[l\left(x_{0}-\right)-l\left(x_{0}\right)\right] \int_{0}^{x_{0}}[h(x)-f(x)] \mu(d x) \\
& \leq 0=\left[l\left(x_{0}\right)-l\left(x_{0}\right)\right] \int_{x_{0}}^{1}[f(x)-h(x)] \mu(d x) \\
& \leq \int_{x_{0}}^{1}\left[l(x)-l\left(x_{0}\right)\right][f(x)-h(x)] \mu(d x),
\end{aligned}
$$

and at least one of the above inequalities is strict. Combining (4.1) nad (4.3), we derive

$$
\int_{0}^{1}\left[l(x)-l\left(x_{0}\right)\right][f(x)-h(x)] \mu(d x)=\int_{0}^{1} l(x)[f(x)-h(x)] \mu(d x)>0,
$$

which is desired conclusion. Clearly, there is no need to subtract $l\left(x_{0}\right)$ in (4.3), if it is non-negative.

Proof of Theorem 1 By (1.6) and (1.7), we can write (1.10) as

$$
d(F, n ; k)=\frac{\int_{0}^{1}\left[F^{-1}(x)\right]^{2} n\left[B_{k, n-1}(x)-B_{k-1, n-1}(x)\right] d x}{\int_{0}^{1} F^{-1}(x) n\left[B_{k, n-1}(x)-B_{k-1, n-1}(x)\right] d x},
$$

$k=1, \ldots, n-1$, and both the integrals are finite.

We first show that the denominator is strictly positive. Note that for all $k=$ $1, \ldots, n-1$ we have

$$
\int_{0}^{1} n B_{k, n-1}(x) d x=\int_{0}^{1} n B_{k-1, n-1}(x) d x=1
$$

and

$$
n\left[B_{k, n-1}(x)-B_{k-1, n-1}(x)\right]\left(x-\frac{k}{n}\right)>0 \text { if } \frac{k}{n} \neq x \in(0,1),
$$


i.e., functions $f=n B_{k, n-1}$ and $h=n B_{k-1, n-1}$ satisfy the assumptions of Lemma 1 with respect to the Lebesgue measure $\mu$. Accordingly, taking $l=F^{-1}$ we show that the statement is true and (4.4) is well-defined.

Observe that $B_{k, n}, k=1, \ldots, n-1$, have the derivatives

$$
B_{k, n}^{\prime}(x)=n\left[B_{k-1, n-1}(x)-B_{k, n-1}(x)\right] .
$$

Owing to Rudin (1964, Theorem 6.30, p. 122), for every $0<\varepsilon<\frac{1}{2}$ we can use the integration by parts formula and write

$$
\begin{aligned}
& \int_{\varepsilon}^{1-\varepsilon}\left[F^{-1}(x)\right]^{2} n\left[B_{k, n-1}(x)-B_{k-1, n-1}(x)\right] d x=-\left[F^{-1}(1-\varepsilon)\right]^{2} B_{k, n}(1-\varepsilon) \\
& \quad+\left[F^{-1}(\varepsilon)\right]^{2} B_{k, n}(\varepsilon)+2 \int_{\varepsilon}^{1-\varepsilon} F^{-1}(x) B_{k, n}(x) F^{-1}(d x) .
\end{aligned}
$$

According to Shiryaev (1996, p. 208), $\mathbb{E} X_{1}^{2}<\infty$ implies that

$$
\lim _{b \rightarrow+\infty} b^{2} F(-b)=\lim _{b \rightarrow+\infty} b^{2}[1-F(b)]=0 .
$$

It follows that

$$
\begin{aligned}
\lim _{\varepsilon \searrow 0}\left[F^{-1}(1-\varepsilon)\right]^{2} B_{k, n}(1-\varepsilon) & =\lim _{b=F^{-1}(1-\varepsilon) \nearrow F^{-1}(1-)} b^{2}\left(\begin{array}{l}
n \\
k
\end{array}\right) F^{k}(b)[1-F(b)]^{n-k}=0, \\
\lim _{\varepsilon \searrow 0}\left[F^{-1}(\varepsilon)\right]^{2} B_{k, n}(\varepsilon) & =\lim _{-b=F^{-1}(\varepsilon) \searrow F^{-1}(0)} b^{2}\left(\begin{array}{l}
n \\
k
\end{array}\right) F^{k}(-b)[1-F(-b)]^{n-k}=0
\end{aligned}
$$

for all $k=1, \ldots, n-1$. Therefore, as $\varepsilon \searrow 0,(4.5)$ takes on the form

$$
\int_{0}^{1}\left[F^{-1}(x)\right]^{2} n\left[B_{k, n-1}(x)-B_{k-1, n-1}(x)\right] d x=2 \int_{0}^{1} F^{-1}(x) B_{k, n}(x) F^{-1}(d x) .
$$

Using similar arguments, we obtain

$$
\int_{0}^{1} F^{-1}(x) n\left[B_{k, n-1}(x)-B_{k-1, n-1}(x)\right] d x=\int_{0}^{1} B_{k, n}(x) F^{-1}(d x),
$$

and finally

$$
d(F, n ; k)=2 \frac{\int_{0}^{1} F^{-1}(x) B_{k, n}(x) F^{-1}(d x)}{\int_{0}^{1} B_{k, n}(x) F^{-1}(d x)} .
$$


Now fix $2 \leq k \leq n-1$, and define

$$
\begin{aligned}
& f(x)=\frac{B_{k, n}(x)}{\int_{0}^{1} B_{k, n}(u) F^{-1}(d u)}=\alpha x^{k}(1-x)^{n-k}, \\
& h(x)=\frac{B_{k-1, n}(x)}{\int_{0}^{1} B_{k-1, n}(u) F^{-1}(d u)}=\beta x^{k-1}(1-x)^{n+1-k},
\end{aligned}
$$

say. Since $F^{-1}$ is non-decreasing, and non-constant, both the integrals are positive, and so are the constants $\alpha$ and $\beta$. Moreover, the integrals of both (4.6) and (4.7) over $[0,1]$ with respect to $F^{-1}$ amount to 1 (cf. (4.1)), and they satisfy (4.2) with $0<x_{0}=\frac{\beta}{\alpha+\beta}<1$. So we apply Lemma 1 with $\mu=l=F^{-1}$, and get

$$
\begin{aligned}
d(F, n ; k) & =2 \int_{0}^{1} \frac{F^{-1}(x) B_{k, n}(x)}{\int_{0}^{1} B_{k, n}(u) F^{-1}(d u)} F^{-1}(d x) \\
& >2 \int_{0}^{1} \frac{F^{-1}(x) B_{k-1, n}(x)}{\int_{0}^{1} B_{k-1, n}(u) F^{-1}(d u)} F^{-1}(d x)=d(F, n ; k-1),
\end{aligned}
$$

which ends the proof.

Acknowledgments The authors are grateful to a referee for valuable comments. The third author was supported by the Polish Ministry of Science and Higher Education Grant no. N N201 416739.

Open Access This article is distributed under the terms of the Creative Commons Attribution Noncommercial License which permits any noncommercial use, distribution, and reproduction in any medium, provided the original author(s) and source are credited.

\section{References}

Arnold BC, Balakrishnan N, Nagaraja HN (1992) A first course in order statistics. J. Wiley, New York Balakrishnan N, Balasubramanian K (1993) Equivalence of Hartley-David-Gumbel and Papathanasiou bounds and some further remarks. Stat Probab Lett 16:39-41

Boland PJ, Samaniego F (2004) The signature of a coherent system and its applications in reliability. In: Soyer R, Mazzuchi T, Singpurwalla N (eds) Mathematical reliability: an expository perspective, international series in operational research and management science. Kluwer, Boston pp 1-29

Castaño-Martinez A, López-Blázquez F, Salamanca-Miño B (2007) Maximal correlation between order statistics. In: Ahsanullah M, Raqab MZ (eds) Recent developments in ordered random variables. Nova Sci. Publ., New York pp 55-68

Jasiński K, Navarro J, Rychlik T (2009) Bounds on variances of lifetimes of coherent and mixed systems. J Appl Probab 46:894-908

Jasiński K, Rychlik T (2011) Maximum variance of order statistics from symmetric populations revisited (submitted)

Johnson NL, Kotz S, Balakrishnan N (1994) Continuous univariate distributions, vol 1, 2nd edn. J. Wiley, New York

Johnson NL, Kotz S, Balakrishnan N (1995) Continuous univariate distributions, vol 2, 2nd edn. J. Wiley, New York

Kochar S, Mukerjee H, Samaniego FJ (1999) The "signature" of a coherent system and its application to comparison among systems. Naval Res Logist 46:507-523 
López-Blázquez F, Salamanca-Miño B (1999) On Terrel's characterization of uniform distribution. Stat Papers 40:335-342

Navarro J, Balakrishnan N (2010) Study of some measures of dependence between order statistics and systems. J Multivar Anal 101:52-67

Navarro J, Rubio R (2010) Computations of coherent systems with five components. Commun Stat Simul Comput 39:68-84

Navarro J, Samaniego FJ, Balakrishnan N, Bhattacharya D (2008) On the application and extension of system signatures to problems in engineering reliability. Naval Res Logist 55:313-327

Papadatos N (1995) Maximum variance of order statistics. Ann Inst Stat Math 47:185-193

Papadatos N (1997) A note on maximum variance of order statistics from symmetric populations. Ann Inst Stat Math 49:117-121

Papadatos N (1999) Upper bound for the covariance of extreme order statistics from a sample of size three. Sankhyā Ser A 61:229-240

Papathanasiou V (1990) Some characterizations of distributions based on order statistics. Stat Probab Lett 9:145-147

Rudin W (1964) Principles of mathematical analysis, 2nd edn. McGraw-Hill, New York

Rychlik T (1994) Distributions and expectations of order statistics for possibly dependent random variables. J Multivar Anal 48:31-42

Rychlik T (2008) Extreme variances of order statistics in dependent samples. Stat Probab Lett 78:15771582

Rychlik T (2011) Applications of Samaniego signatures to bounds on variances of coherent and mixed systems. In: Lisnianski A, Frenkel I (eds) Recent advances in system reliability: signatures, multi-state systems and statistical inference. Springer, London (to appear)

Samaniego F (1985) On closure of the IFR class under formation of coherent systems. IEEE Trans Reliab TR-34:69-72

Shaked M, Suarez-Llorens A (2003) On the comparison of reliability experiments based on the convolution order. J Am Stat Assoc 98:693-702

Shiryaev AN (1996) Probability, 2nd edn. Springer, New York

Székely GJ, Móri TF (1985) An extremal property of rectangular distributions. Stat Probab Lett 3:107-109

Terrel GR (1983) A characterization of rectangular distributions. Ann Stat 11:823-826 